\title{
Influence of the drug distribution in electrospun gliadin fibers on drug-release behavior
}

\author{
Ying $\mathrm{Xu}^{1, \mathrm{a}}$, Jiao-Jiao $\mathrm{Li}^{1, \mathrm{a}}$, Deng-Guang $\mathrm{Yu}^{1, *}$, Gareth R. Williams ${ }^{2}$, \\ Jun-He Yang ${ }^{1}$, Xia Wang ${ }^{1, *}$, \\ ${ }^{1}$ School of Materials Science \& Engineering, University of Shanghai for Science and \\ Technology, Shanghai 200093, China \\ ${ }^{2}$ UCL School of Pharmacy, University College London, 29-39 Brunswick Square, \\ London WC1N 1AX, UK.
}

* Corresponding authors:

Prof. Deng-Guang Yu

Phone: +86 2155270632

Fax: +862155270632

E-mail : ydg017@usst.edu.cn

Prof. Xia Wang

E-mail: wangxia@usst.edu.cn

${ }^{a}$ These authors contributed equally. 


\section{ABSTRACT}

Drug distribution within its carrier in a solid dosage form often generates a profound influence on its release profile, particularly when the physicochemical properties of the carrier are exploited to manipulate drug release behavior. In this job, two different types of distributions of a model drug ibuprofen (IBU) within a protein gliadin in their electrospun nanofibers were intentionally created. One was homogeneous distribution in the monolithic fibers fabricated using a modified coaxial process, and the other one was heterogeneous distribution in the core/shell fibers prepared through a traditional coaxial process. SEM observations clearly demonstrated the different distributions of IBU within gliadin in the two kinds of nanofibers although both of them had smooth surfaces and linear morphology. XRD results showed that IBU was amorphously distributed in the monolithic fibers, but that some IBU crystalline lattices presented in the core/shell fibers. FTIR and RM spectra suggested that gliadin had good compatibility with IBU. In vitro dissolution tests verified that the gliadin nanofibers with a heterogeneous drug distribution could provide a better sustained release profile than its counterpart in terms of initial burst release and sustained release time period. Both the fiber formation and drug-controlled release mechanisms are suggested. The present study demonstrated a concept that drug distribution with the medicated nanomaterials can be exploited as a tool to optimize the drug sustained release profile.

Keywords: Gliadin, coaxial electrospinning, drug-loaded fibers, sustained release, drug distribution

\section{Chemical compounds studied in this article}

Gliadin (PubChem CID: 17787981); Ibuprofen (PubChem CID: 3672); 1,1,1,3,3,3-Hexafluoro-2-propanol (PubChem CID:13529); Ethanol (PubChem CID: 702); Trifluoroacetic acid (PubChem CID:6422); Trifluoroethanol (PubChem CID:6409). 


\section{Introduction}

Conventional drug delivery systems (DDSs) potentially exhibit an uncontrollable initial burst of release, which results in the oscillation of the systemic drug concentration, leading to both under- and overdosing. A much better therapeutic outcome is usually achieved if the drug can be maintained at a constant concentration in the body (Kazemimostaghim et al., 2015; Kim et al., 2016); in this case, the sustained release of the drug from a DDS is often required for optimum therapeutic effects (Paliwal and Palakurthi, 2014). Sustained (or extended) release DDSs free the loaded drug over a prolonged duration, providing a close to constant systemic concentration. By ensuring that this concentration remains within the therapeutic window, they provide high therapeutic efficacy with minimum side effects (Babić, 2015). As they reduce the dosage interval required for successful treatment, oral sustained-release DDSs tend to have good patient compliance (Liu and Feng, 2015). For this reason, materials and methods providing sustained drug release profiles have been widely investigated, with a vast array of reports in the literature. A wide range of formulation types and carriers have been explored, such as Eudragit RLPO® nanoparticles prepared by nanoprecipitation (Gandhi et al., 2015), drug loaded in spherical and tubular nanocarriers via layer-by-layer (LbL) encapsulation (Shutava et al., 2014), TC(tetracycline) loaded onto Ag@SiO2-MIP (molecularly imprinted polymers) (AguilarAarcía et al., 2016), gelatin-montmorillonite nanoparticles prepared by desolvation (Sarmah et al., 2015), microparticles prepared by spray-drying method and polymeric nanofibers fabricated using electrospinning 
(Sóti et al., 2015) .

When developing new drug delivery systems, considering both the type of material and the physical properties of the formulation (e.g. its size, structure and shape) is important. The reason is that all these factors can have a significant influence on the release profile of the drug (Peltonen et al., 2010). Selecting a carrier material that is biocompatible is also vital (Kazemimostaghim et al., 2015). To this end, natural polymers, such as gliadin, have attracted much research attention.

Proteins are popular in developing new kinds of nano drug delivery systems for poorly water-soluble drugs (He et al., 2013; Bohr et al., 2014; Babitha et al., 2017). Gliadin is a plant protein with good biocompatibility and biodegradability (Gulfam et al., 2012). Being derived from natural sources, it does not suffer from the presence of monomer or initiator residues, which can bring problems to the synthetic materials (Elzoghby et al., 2012). The use of plant protein is also more "environmentally economical" compared with animal-derived proteins (Wan et al., 2015). Gliadin has been studied extensively: for instance, fibers with excellent mechanical property and water stability have been prepared using wet spinning (Reddy and Yang, 2008), and nanoparticles for food-grade colloidal delivery systems have been reported (Joye et al., 2015). The application of gliadin microspheres as carriers for drug/nutrient delivery have been explored further (Wan et al., 2015). Although there exist several reports on the electrospinning of gliadin, in which acid or basic solutions have been explored as working fluids with the help of additives, such as polyhedral oligomeric silsesquioxane (Soares et al., 2011; Wang et al., 2012), no report has yet to explore the applications of electrospun gliadin fibers in drug delivery to the best of our 
knowledge. According to the most recent investigations, these medicated gliadin fibers have the potentials to be developed into commercial tablets or capsules (Démuth et al., 2016; Illangakoon et al., 2015).

Electrospinning has evoked considerable interest as a simple, versatile, and economical method to produce polymer fibers and polymer-based nanocomposites (Rosic et al., 2012; Yu et al., 2017; Yang et al., 2017). The electrospun fibers often have micro- to nano-meter sizes with a series of unique characteristics, such as a large surface area to volume ratio and high porosity (Ji et al., 2013). In virtue of these characteristics, electrospun fibers have been broadly investigated for potential applications in a wide variety of fields. These include, for instance, removing heavy metals from wastewater (El-Sherif et al., 2013; Taha et al., 2012; Wen et al., 2016), tissue engineering ( $\mathrm{Ji}$ et al., 2013; Wang et al., 2013), photocatalytic degradation (Choi et al., 2015; Pascariu et al., 2016), thermal energy storage (Chen et al., 2013), supercapacitor electrodes (Tolosa et al., 2016), solar cells (Jin et al., 2014), lithium-ion batteries (Han et al., 2016; Zhu et al., 2016), drug delivery (Borbás et al., 2016; Paaver et al., 2015; Seif et al., 2015), and military protective clothing (Gorji et al., 2012).

In terms of biomedical applications, electrospun fibers allow the production of sophisticated nanostructures with control of the fiber alignment, porosity, and size possible. This has led to a range of new polymer-based DDSs (Rasekh et al., 2015; Yang et al., 2016; Yu et al., 2015). However, obtaining high quality medicated fibers using a simple single-fluid electrospinning process remains difficult, because a series of parameters can affect the process. For example, many pharmaceutical polymers have a very narrow electrospinnable concentration window, which depends on both the polymer and solvent characteristics (Pelipenko et al., 2015). In the literature, many 
medicated fibers have been fabricated from a co-dissolving solution of a guest drug and a host polymer using a single-fluid or coaxial process (Illangakoon et al., 2015; Wang et al., 2015; Xu et al., 2015). In these monolithic products, the drug is typically homogeneously distributed throughout the resultant fibers. Often, the achievement of a sustained-release profile depends mainly on the physicochemical properties of the filament-forming polymer matrix with little considerations about the drug distribution within the nanofibers. However, drug distributions within the DDSs should have a deep influence on its functional performance regardless of traditional dosage forms (Muehlenfeld et al., 2013; Punčochová et al., 2016; Schrank et al., 2015; Vukosavljevic et al., 2016; Windbergs et al., 2010) or advanced nano DDSs (Hu et al., 2016; Huang et al., 2016; Kim et al., 2016; Saeidpour et al., 2017).

In this work, single-fluid electrospinning was first conducted to determine the electrospinnable concentration window of gliadin. Later, both traditional and modified coaxial electrospinning processes were carried out to tailor the within-fiber drug distribution intentionally. Ibuprofen (IBU) was exploited as the model drug because it is one of the most commonly used non-steroidal anti-inflammatory drugs (NSAIDs), it has a large consumption all over the world, and it is a typical poorly water-soluble drug utilized as a model in literature (Li et al., 2013; Zhang et al., 2017; Shen et al., 2017). Thus, it would be a suitable representative for investigating the influence of drug distribution on its release behavior. The fibers were characterized in terms of their morphology, nanostructures, physical forms of components, and drug sustained-release profiles.

\section{Materials and methods}

\subsection{Materials}

IBU was obtained from the Hubei Biocause Pharmaceutical Co., Ltd. (Hubei, China). 
1,1,1,3,3,3-Hexafluoro-2-propanol (HFIP, purity 99.0\%), wheat gliadin (extracted from wheat), anhydrous ethanol, trifluoroacetic acid, and trifluoroethanol were provided by the Sinopharm Chemical Reagent Co., Ltd. (Shanghai, China). Water was double distilled before use.

\subsection{Electrospinning}

Two syringe pumps (KDS100 and KDS200, Cole-Parmer, Vernon Hills. IL, USA) and a ZGF2000 high-power supply (60 kV/2 mA, Shanghai Sute Corp., Shanghai, China) were used for all electrospinning experiments, together with an in-house coaxial spinneret. Fibers were collected on a flat piece of cardboard covered with Al foil.

\subsubsection{Gliadin electrospinnability}

Gliadin solutions with concentrations of $5,10,15,20$ and $25 \%(w / v)$ were prepared by adding appropriate amounts of gliadin powder to HFIP and stirring until homogeneous solutions were produced. The pump used to drive the sheath liquid was switched off for these experiments, and the liquids were pumped through the core channel only. Electrospinning was conducted under ambient conditions $\left(22{ }^{\circ} \mathrm{C} \pm 5{ }^{\circ} \mathrm{C}\right.$, and relative humidity of $58 \% \pm 6 \%$ ). After some initial optimization, the distance between the tip of the spinneret and the collector, the applied voltage, and the flow rate were fixed at $14 \mathrm{~cm}, 15 \mathrm{kV}$, and $2.5 \mathrm{~mL} / \mathrm{h}$, respectively.

\subsubsection{Coaxial electrospinning}

For the successful implementation of a coaxial electrospinning processes, only one of the two working fluids must be electrospinnable (Huang et al., 2006). Although the spinnable sheath fluid is traditionally required, many reports using a spinnable core and unspinnable sheath (commonly called "modified coaxial electrospinning") can be found in the literature (Wang et al., 2015). Specifically, pure solvents can be 
utilized as sheath fluids to help prevent clogging and ensure the production of high quality fibers (Yu et al., 2012).

In the traditional coaxial processes, an IBU solution in HFIP was used as the core fluid, with a gliadin sheath solution. In the modified process, HFIP formed the sheath fluid, and a mixed gliadin/IBU solution comprised the core. After some optimization, the core-to-sheath fluid flow rate ratios were fixed at $0.5: 2.5 \mathrm{~mL} / \mathrm{h}$ and $2.5: 0.5 \mathrm{~mL} / \mathrm{h}$ in the traditional and modified coaxial processes, respectively. The applied voltage was fixed at $15 \mathrm{kV}$, and the fibers were collected on aluminum foil at a distance of 14 $\mathrm{cm}$ from the tip of the spinneret (see Table 1). The compositions of the working fluids for the coaxial processes are also listed in Table 1.

\subsection{Characterization}

\subsubsection{Morphology}

The surface and cross-section morphology of the electrospun products were assessed using a Quanta 450 FEG field emission scanning electron microscope (FESEM; FEI Inc., Hillsboro, OR, USA). The samples were gold sputter-coated under a nitrogen atmosphere before examination. Images were then recorded at an excitation voltage of $20 \mathrm{kV}$. The samples of cross-sections were prepared by immersing a strip of non-woven mats into the liquid nitrogen for over 20 minutes, followed by the manual breaking of the strip. The fiber diameters were estimated using ImageJ software on the SEM images and over 100 places.

\subsubsection{Physical form}

X-ray diffraction (XRD) patterns were obtained using a D8 Advance diffractometer (Bruker Corporation, Karlsruhe, Germany) over the $2 \theta$ range of $5^{\circ}-$ $60^{\circ}$ at $8^{\circ} / \mathrm{min}$. The instrument was supplied with $\mathrm{Cu} \mathrm{K \alpha}$ radiation $(\lambda=1.5418 \AA)$ at 40 $\mathrm{mV}$ and $40 \mathrm{~mA}$. 


\subsubsection{IR and Raman spectroscopy}

Attenuated total reflectance Fourier transform infrared (ATR-FTIR) analysis was conducted using a Spectrum 100 FTIR spectrometer (Perkin Elmer, Waltham, MA, USA). The scanning range was $500 \mathrm{~cm}^{-1}-4000 \mathrm{~cm}^{-1}$, with a resolution of $2 \mathrm{~cm}^{-1}$. Raman spectra were obtained at room temperature, using a LabRAM HR Evolution spectrometer (Horiba Scientific, Longjumeau, France). Samples were placed on microscope slides, and Raman spectra were recorded at least three different positions, over the range of 300 to $3000 \mathrm{~cm}^{-1}$. Excitation was at $532 \mathrm{~nm}(\mathrm{He} / \mathrm{Ne}$ laser, $<10$ $\mathrm{mW})$.

\subsection{In vitro drug dissolution tests}

In vitro release tests were conducted in $50 \mathrm{~mL}$ phosphate buffer solution (PBS, $\mathrm{pH}=6.8,0.2 \mathrm{~mol} / \mathrm{L}$ ) with an amount of fibers containing $1.0 \mathrm{mg}$ IBU (i.e. $16 \mathrm{mg}$ of M1 and E1, and $8.5 \mathrm{mg}$ of M2 and E2). All experiments were carried out in a shaking incubator (BDY-200D, Baidian Scientific Instrument Co. Ltd., Shanghai, China) at a constant temperature at $37{ }^{\circ} \mathrm{C}$ and a rotation rate of $100 \mathrm{rpm}$. At predetermined time points, $2.0 \mathrm{~mL}$ aliquots were withdrawn and replaced by an equal volume of PBS to maintain a constant volume. After filtration through a membrane with pore size of 0.45 $\mu \mathrm{m}$ (Sinopharm Chemical Reagent Co., Ltd, Shanghai, China), samples were assayed at 264 nm using a Lambda 750S UV-vis spectrophotometer (Perkin Elmer, Waltham, MA, USA). The concentration of IBU was calculated based on a predetermined calibration curve. All measurements were conducted six times and were reported as mean \pm S.D.

\section{Results and discussion}

\subsection{The gliadin electrospinnable window}

The most important factors in the preparation of electrospinnable working fluids are the selection of solvent and the polymer concentration. Often, polymers have a 
very narrow electrospinnable concentration window in a particular solvent. Gliadin could be electrospun into fibers from its acid or basic solutions. However, the use of acid or basic conditions is undesirable because of the risk of protein degradation. In this work, we sought to process gliadin without using potentially detrimental additives. A panel of solvents (including HFIP, anhydrous ethanol, trifluoroacetic acid, trifluoroethanol and their mixture) was explored for their ability to dissolve gliadin, and HFIP was determined to be the most suitable one because of its low boiling point $\left(59{ }^{\circ} \mathrm{C}\right)$ and the fine solubility and stability of gliadin in it. A series of gliadin solutions with varied concentrations of $5,10,15,20$ and $25 \% \mathrm{w} / \mathrm{v}$ were prepared for electrospinning. The results are given in Fig. 1.

No fibers were generated from the solution containing 5\% gliadin, because at such a low concentration, there were insufficient physical chain entanglements to form these. Thus, the solution underwent a typical electrospraying process, with microparticles as the final products (Fig. 1a). These particles had a size of several microns and a dimpled morphology. The latter arose because, during electrospraying, the surface of the droplets solidifies to form a rigid membrane, with some unevaporated solvents remaining. Further evaporation of the solvents causes the particles to collapse on themselves.

The gliadin solution with a concentration of $25 \%$ was too viscous to be drawn by the electrical forces, and the spinneret was frequently blocked by semi-solid substance forming around it. Nevertheless, the electrospinning of solutions containing 10, 15 and $20 \%$ gliadin could be undertaken relatively continuously and smoothly, and only an occasional manual removal of semi-solid materials from around the spinneret was required. The products are shown in Figs. 1b, c and d, respectively. As can be seen, a concentration of $10 \%$ (Fig. 1b) was still too low to support the formation of linear 
fibers, and the products had beads-on-a-string or spindles-on-a-string morphologies. In contrast, the gliadin solutions at $15 \%$ (Fig. 1c) and 20\% (Fig. 1d) gave fibers with free of beads and spindles. The former had a smaller and narrower distribution diameter $(0.72 \pm 0.21 \mu \mathrm{m})$ than the latter $(1.76 \pm 0.53 \mu \mathrm{m})$. Overall, gliadin has an electrospinnable concentration window between $15 \%$ to $20 \% \mathrm{w} / \mathrm{v}$ when HFIP is used as the solvent.

\subsection{Morphology of the IBU-loaded gliadin fibers}

The raw IBU particles have a typical crystalline morphology, comprising short rods (Fig. 2a), while the raw gliadin powder having a flat globular shape (Fig. 2b). The SEM images of the drug-loaded fibers' surfaces consisting of IBU and gliadin are shown in Fig. 2c to 2f. Fibers M1 and M2 from the modified coaxial processes (Figs. $2 \mathrm{c}$ and $2 \mathrm{~d}$ ) had smooth surfaces and linear morphology without any beads-on-a-string phenomenon. They had narrow diameter distributions of $0.92 \pm 0.13 \mu \mathrm{m}$ and $0.89 \pm$ $0.14 \mu \mathrm{m}$, respectively. In contrast, the core/shell fibers E1 and E2 from the traditional coaxial processes had two distinct fiber populations (Figs. 2e and 2f). Many extremely fine fibers can be observed, along with population sizes of $0.93 \pm 0.42 \mu \mathrm{m}$ and $1.00 \pm$ $0.37 \mu \mathrm{m}$ for E1 and E2, respectively. Similar phenomena can be found in the pure gliadin fibers that were prepared using single-fluid electrospinning, although these occurred to a lesser extent. These phenomena suggest that the modified coaxial processes help create higher quality fibers in terms of fiber size and size distribution.

The fibers M1 and M2 from the modified coaxial processes had a diameter slightly smaller than E1 and E2, which were formed under the traditional coaxial processes. This result can be attributed to the use of a pure solvent as the sheath fluid in the modified process. This sheath solvent can slow down or even prevent the premature formation of semi-solid substances on the surface of the working fluid jets, 
which in turn, helps the jets to be drawn and dried homogeneously for a longer time period. The presence of a solvent layer surrounding the working polymer solution can also weaken some negative influences of the surrounding environment and spinneret on the fluid jets.

The SEM images of the drug-loaded fibers' cross-sections are shown in Fig. 3. Just as anticipated, the cross-sections of fibers M1 and M2 (Figs. 3a and 3b and their upper-right insets, respectively) were smooth without any discernible nanoparticles resulting from the solid phase separation. In sharp contrast, the cross-sections of fibers E1 and E2 (Figs. 3c and 3d and their upper-right insets) had a central region. The central regions had many tiny particles within them, giving a hint about the possible re-crystallization of IBU nanoparticles from the core pure IBU working solutions during the traditional coaxial electrospinning processes. This result suggests that the fibers E1 and E2 from the traditional coaxial processes had the core-shell nanostructures with the drug being heterogeneously distributed into the gliadin fibers.

\subsection{Physical form}

XRD analysis (Fig. 4) was undertaken to determine the physical form of IBU in the drug-loaded fibers. Numerous distinct reflections in the XRD pattern of raw IBU (Fig. 4a) can be observed, confirming it to be a crystalline material. Meanwhile, pure gliadin is amorphous, displaying no Bragg reflections and only broad humps in its pattern (Fig. 4b).

In the XRD patterns of the monolithic fibers M1 and M2 (Figs. 4c and 4d), the characteristic Bragg reflections of IBU were absent. Thus, the IBU loaded in the fiber had been fully converted into the amorphous state by the electrospinning process. In contrast, several characteristic reflections of IBU were detected in the XRD patterns of E1 and E2 (Figs. 4e and 4f), which were superimposed on an amorphous 
background. Hence, as can be clearly seen, at least a portion of the IBU in the fibers exists in the crystalline state. During the preparation of E1 and E2, the core fluids were pure IBU solutions of HFIP. After the evaporation of the solvent molecules, the IBU molecules within the gliadin sheath presumably re-crystallized to minimize the energy of the system. This could possible for the E fibers because there were no polymer molecules in the core hindering this process, but not for the $\mathrm{M}$ fibers where the drug and polymer were intimately mixed; thus, the gliadin molecules provided steric hindrance to recrystallization.

\subsection{Component compatibility}

Compatibility among their components is important not only for the formation of composite fibers, but also for their long-term stability. Second-order interactions, such as hydrogen bonding, hydrophobic interactions, and electrostatic forces between the drug and polymer should improve compatibility, and can be investigated by FTIR and Raman spectroscopy. The FTIR spectra of the raw materials and their fibers are given in Fig. 5a. The chemical structures of IBU and gliadin are shown in Fig. 5b.

In the IR spectrum of pure IBU (Fig. 5a), numerous vibrations are visible: the absorbance at $2957 \mathrm{~cm}^{-1}$ was assigned to the stretching of the O-H group, that at 1713 $\mathrm{cm}^{-1}$ corresponded to the stretching of the $\mathrm{C}=\mathrm{O}$ group, whereas the peak at $1230 \mathrm{~cm}^{-1}$ was due to C-O group stretching. Peaks at 1508, 1462, 1420, and $778 \mathrm{~cm}^{-1}$ can be attributed to the presence of a phenyl group. Gliadin showed a wide absorption band centered at $3290 \mathrm{~cm}^{-1}$, assigned to the stretching of the H-bonded O-H groups of the amino acid present in gliadin; two bands of different intensities of 1652 and $1544 \mathrm{~cm}^{-1}$ were associated with the band vibrations of the $\mathrm{C}=\mathrm{O}$ and $\mathrm{C}-\mathrm{N}$ groups, respectively.

Comparing the spectra of the fibers M1, M2, E1 and E2 with that of raw IBU, the IBU phenyl absorption peaks at $1508,1462,1420$, and $778 \mathrm{~cm}^{-1}$ disappeared, as 
did the carboxylate peak at $1713 \mathrm{~cm}^{-1}$ in the majority of cases. Considering the molecular structures of the two fiber components (Fig. 5b), it can be suggested that hydrogen bonds may have formed between them. The IBU and gliadin molecules possess free hydroxyl groups and amino groups, which can act as proton donors, as well as carbonyl groups that can serve as proton receptors for forming hydrogen bonds (Fig. 5c). This explains the observations seen for the modified coaxial fibers M1 and M2.

However, the characteristic peaks of IBU were also not visible in the spectra of E1 and E2, despite the fact that the XRD indicated the presence of crystalline IBU in these systems. The failure to achieve signals of IBU re-crystallized nanoparticles using ATR-FTIR could be attributed to the fact that only a small proportion of the IBU molecules in the core parts of fibers had re-crystallized, which was too small and too far away from the fiber surfaces to be detected using the FTIR instrument.

Raman spectra were collected in an attempt to unravel these factors. Results are shown in Fig. 6. As can be seen, the pure IBU spectrum had peaks at $2719(\mathrm{O}-\mathrm{H}$ stretching) and 1608, 1463, 1206, 1180, and $1115 \mathrm{~cm}^{-1}$ (corresponding to the phenyl group), all of which were absent in the spectra of the fibers, thus confirming the presence of intermolecular interactions (most likely hydrogen bonding) between the IBU and gliadin. Similar to the IR data, the Raman spectra of E1 and E2 showed no evidence for the presence of IBU crystals, thus confirming that this phenomenon occurred because only a small proportion of the total drug loading recrystallized (the Raman probe had a much greater penetration depth than ATR-FTIR).

\subsection{In vitro drug release profiles}

The drug release profiles of the fibers are given in Fig. 7a. M1 and M2 showed a distinct initial burst of release, freeing $39.60 \%$ and $38.47 \%$ of the incorporated drug 
respectively in the first $30 \mathrm{~min}$, respectively. In contrast, E1 and E2 released only $12.59 \%$ and $13.20 \%$ of their IBU loading in the same period, respectively, thus demonstrating a much reduced burst effect. After the experiment had been running for $8 \mathrm{~h}, \mathrm{M} 1$ and $\mathrm{M} 2$ released $92.81 \%$ and $92.61 \%$ of the loaded IBU, respectively, after which the release profile tailed off. E1 and E2 could sustain drug release much more effectively, respectively reaching $93.66 \%$ and $92.42 \%$ release after $16 \mathrm{~h}$ before tailing-off occurred.

To investigate the drug release mechanism, data were analyzed using the Peppas equation, $\mathrm{Q}=\mathrm{kt}^{\mathrm{n}}$ (Peppas, 1985). In this model, $\mathrm{Q}$ is the percentage of drug released, $\mathrm{t}$ is the time, $\mathrm{k}$ is the rate constant, and $\mathrm{n}$ is the release exponent, indicative of the drug release mechanism. The regressed equations for $\mathrm{M} 1$ and $\mathrm{M} 2$ between $0 \mathrm{~h}$ and $8 \mathrm{~h}$ were $\mathrm{Q}_{\mathrm{M} 1}=50.00 \mathrm{t}^{0.31}\left(\mathrm{R}^{2}=0.9924\right)$ and $\mathrm{Q}_{\mathrm{M} 2}=50.21 \mathrm{t}^{0.30}\left(\mathrm{R}^{2}=0.9918\right)$, respectively. Both the $\mathrm{n}$ values were less than 0.45 , indicating that IBU release from M1 and M2 was controlled by a typical Fickian diffusion mechanism. Meanwhile, the results for E1 and E2 were $\mathrm{Q}_{\mathrm{E} 1}=21.84 \mathrm{t}^{0.53}\left(\mathrm{R}^{2}=0.9926\right)$ and $\mathrm{Q}_{\mathrm{E} 2}=23.02 \mathrm{t}^{0.51}\left(\mathrm{R}^{2}=0.9904\right)$, respectively. These $\mathrm{n}$ values were greater than 0.45 , suggesting an apparent diffusion/erosion mechanism was operational in these systems.

To further compare the release properties of the fibers, the times taken for the release of $30 \%, 50 \%$, and $80 \%$ of the contained IBU were calculated from their Peppas model (Fig. 7b). Although the drug contents in the E2 and M2 fibers were about 1.9-fold higher compared with those in E1 and M1 fibers, the drug release behaviors from fibers E1 and E2 were similar as well as those from M1 and M2. These phenomena suggest that the drug contents have almost no influence on the drug release behaviors. This is because fibers M1 and M2 have the same drug-loaded host matrix (gliadin), the similar fibers' diameters and homogeneous drug distribution, and 
the same drug diffusion mechanisms. Similarly, E1 and E2 have a similar drug release behavior. However, fibers M1 and E1 (and also M2 and E2) resulted in significantly different drug-release behaviors even though they have the same drug contents. Hence, the different nanostructures and the correspondingly different drug distributions have played their roles in manipulating the drug release behaviors.

\subsection{Fiber formation and drug release mechanisms}

Although the fibers from the modified coaxial processes have better quality than those from the traditional coaxial processes in terms of their fiber sizes and size distributions, the latter provided better functional performance considering their drug sustained-release profiles. The drug distributions within the fibers and also their physical forms contributed to the different release behaviors observed. The modified coaxial process ensured a smooth and robust preparation process, but resulted in monolithic fibers with a homogeneous distribution of amorphous IBU in the gliadin matrix, which in turn resulted in a worse sustained release profile with a more severe initial burst release effect and a shorter drug release time period.

By taking advantage of the interactions between the electronic energy and working fluids, electrospinning processes can solidify the fluids within several decades of milliseconds regardless of how many working fluids simultaneously. During this extremely fast process, the physical state of components in the working fluids can be propagated into the solid products without solid phase separations. Provided the favorable secondary interactions (such as hydrogen bonds, hydrophobic and electrostatic interactions) exist between the drug and carrier, there should be no chances for the drug molecules to migrate, to meet and to re-crystallize into crystalline lattices. Thus here, the modified coaxial process can amorphize IBU from its co-dissolving solution with gliadin to result in a monolithic composite with a 
uniform drug distribution all over the whole fibers.

In contrast, in the traditional coaxial processes the drug was confined inside an insoluble gliadin sheath layer, which resulted from the use of a drug solution as the core working fluid. In coaxial electrospinning processes, generally little diffusion happens between the core and shell fluids owing to the extremely fast drawing and drying processes (Yang et al., 2016). However, some drug molecules may have migrated from the core to the sheath fluid during the traditional coaxial processes, which has been demonstrated in some electrosprayed products (Bohr et al., 2015; Wan et al., 2014). These migrations should result in the release of some drug molecules at the very early stages of the experiment.

Fig. 8a schematically depicts the proposed fiber formation mechanisms in the traditional coaxial processes. In the bending and whipping region, Coulombic repulsion $\left(f_{\mathrm{C}}\right)$ from the surface charges on the fluid jets is responsible for the drawing and thinning of the viscous gliadin solution. The drug molecules may have possibly moved along the direction of the electric field $\left(f_{\mathrm{E}}\right)$, which is perpendicular to the Coulombic drawing. This phenomenon is likely to be aided by the concentration gradient, given the presence of a concentrated IBU core solution and lack of drug in the sheath fluid. This would result in some amount of drug being present in the shell of the fibers. Owing to the favorable interactions between the drug and polymer, any IBU in the shell would be expected to exist in the amorphous physical form. In contrast, the IBU in the core can potentially re-crystallize, as demonstrated by the XRD data in Fig. 4.

A schematic diagram of the proposed drug distribution in the fibers from the two spinning processes is exhibited in Fig. 8b. M1 and M2 are monolithic fibers with a homogeneous IBU distribution. The drug release mechanism is a typical Fickian 
diffusion mechanism. When they are added to the dissolution medium, water molecules gradually penetrate into the fibers; then, the IBU molecules dissolve into this water and permeate out into the bulk dissolution medium. Owing to the amorphous nature of the IBU, the uniform drug distribution, the large surface area, and the short diffusion distance for drug molecules close to the exterior of the fibers, an initial burst release is inevitable.

However, fibers E1 and E2 have a heterogeneous distribution of IBU, where the drug is concentrated in the core with a minority of molecules proposed to be present in the shell. The drug release mechanism is thought to rely both on diffusion and erosion based on the Peppas model, although it should be noted that this model assumes a uniform distribution of drug throughout the matrix, and thus its results must be taken with caution here. Given that gliadin is insoluble in water, the IBU can only be released by diffusion through the fiber shell. The presence of only a small amount of drug in the shell and the formation of IBU crystals in the core result in a much slower rate of release for these core/shell systems than for the monolithic fibers. The differences of drug stability between the monolithic amorphous fibers and the heterogeneous core/shell fibers with some drug crystalline lattices deserve to be further investigated.

\section{Summary and conclusions}

The electrospinnability of gliadin was investigated, and its electrospinnable concentration window in HFIP was successfully determined to be between $15 \%$ and $20 \%$ w/v. With gliadin as drug carrier, two kinds of IBU-loaded fibers were fabricated using two types of coaxial electrospinning processes. SEM observations showed that all these fibers had linear morphology with smooth surfaces, but the fibers from 
traditional coaxial process had a heterogeneous drug distribution in their core/shell structures, whereas those from the modified coaxial process possessed a homogeneous drug distribution in their monolithic structures. The different physical forms of IBU in the different distributions were demonstrated by XRD tests, one was totally amorphous and homogeneous, and the other was detected to have IBU crystalline lattices. FTIR and RM spectra suggested that the drug and carrier had good compatibility. In vitro dissolution tests verified that the gliadin fibers with a heterogeneous drug distribution had fewer initial burst drug release and a longer time period release of $16 \mathrm{~h}$, suggesting a better sustained drug release profile than those fibers having a homogeneous drug distribution who had severe initial burst release and a shorter release time period of $8 \mathrm{~h}$. The different drug distributions have manipulated the different release behaviors of the loaded drug molecules, and thus resulted in different drug sustained release profiles.

\section{ACKNOWLEDGEMENTS}

This study was supported by the National Natural Science Foundation of China (No. 51373101) and the Key Project of Basic Research in Shanghai (No. 15JC-1490700).

\section{References}

AguilarAarcía, D., OchoaTrán, A., ParaguayDelgado, F., DíazGarcía, M.E., PinaLuis, G., 2016. Water-compatible core-shell Ag@SiO2 molecularly imprinted particles for the controlled release of tetracycline. J. Mater. Sci. 51, 5651-5663. 
Babić, M.M., Antić, K.M., Vuković, J.S.J., Božić, B.D., Davidović, S.Z., Filipović, J.M., Tomić, S.L., 2015. Oxaprozin/poly(2-hydroxyethyl acrylate/itaconic acid) hydrogels: morphological, thermal, swelling, drug release and antibacterial properties. J. Mater. Sci. 50, 906-922.

Babitha, S., Rachita, L., Karthikeyan, K., Shoba, E., Janani, I., Poornima, B., Sai, K. P., 2017. Electrospun protein nanofibers in healthcare: A review. Int. J. Pharm. $523,52-90$.

Bohr, A., P Boetker, J., Rades, T., Rantanen, J., Yang, M., 2014. Application of spray-drying and electrospraying/electospinning for poorly watersoluble drugs: a particle engineering approach. Curr. Pharm. Design 20, 325-348.

Bohr, A., Wan, F., Kristensen, J., Dyas, M., Stride, E., Baldursdottír, S., Edirisinghe, M., Yang, M., 2015. Pharmaceutical microparticle engineering with electrospraying: the role of mixed solvent systems in particle formation and characteristics. J. Mater. Sci. Mater. Med. 26, 61.

Borbás, E., Sinkó, B., Tsinman, O., Tsinman, K., Kiserdei, E., Démuth, B., Balogh, A., Bodák, B., Domokos, A., Dargó, G., Balogh, G.T., Nagy, Z.K., 2016. Investigation and mathematical description of the real driving force of passive transport of drug molecules from supersaturated solutions. Mol. Pharm. 13, 3816-3826.

Chen, C.Z., Zhao, Y.Y., Liu, W.M., 2013. Electrospun polyethylene glycol/cellulose acetate phase change fibers with core-sheath structure for thermal energy storage. 
Renew. Energ. 60, 222-225.

Choi, W.S, Choi, I.S., Lee, J.K., Yoon, K.R., 2015. Preparation of fluorescein-functionalized electrospun fibers coated with $\mathrm{TiO} 2$ and gold nanoparticles for visible-light-induced photocatalysis. Mater. Chem. Phys. 163, 213-218.

Démuth, B., Nagy, Z.K., Balogh, A., Vigh, T., Marosi, G., Verreck, G., Van Assche, I., Brewster, M.E., 2015. Downstream processing of polymer-based amorphous solid dispersions to generate tablet formulations. Int. J. Pharm. 486, 268-286.

El-Sherif, I.Y., Tolani, S., Ofosu, K., Mohamed, O.A., Wanekaya, A.K., 2013. Polymeric nanofibers for the removal of $\mathrm{Cr}(\mathrm{III})$ from tannery waste water. J. Environ. Manage. 129, 410-413.

Elzoghby, A.O., Samy, W.M., Elgindy, N.A., 2012. Protein-based nanocarriers as promising drug and gene delivery systems. J. Control. Release 161, 38-49.

Gandhi, A., Jana, S., Sen, K.K., 2014. In-vitro release of acyclovir loaded Eudragit RLPO® nanoparticles for sustained drug delivery. Int. J. Biol. Macromol. 67, 478-482.

Gorji, M, Jeddi, A.A.A., Gharehaghaji, A.A., 2012. Fabrication and characterization of polyurethane electrospun nanofiber membranes for protective clothing applications. J. Appl. Polym. Sci. 125, 4135-4141.

Gulfam, M., Kim, J.E., Lee, J.M., Ku, B., Chung, B.H., Chung, B.G., 2012. 
Anticancer drug-loaded gliadin nanoparticles induce apoptosis in breast cancer cells. Langmuir 28, 8216-8223.

Han, P., Yuan, T., Yao, L., Han, Z., Yang, J.H., Zheng, S.Y., 2016. Copper nanoparticle-incorporated carbon fibers as free-standing anodes for lithium-ion batteries. Nanoscale Res. Lett. 11, 172.

He, W., Lu, Y., Qi, J., Chen, L., Hu, F., Wu, W., 2013. Food proteins as novel nanosuspension stabilizers for poorly water-soluble drugs. Int. J. Pharm. 441, 269-278.

Hu, Q., Sun, W., Lu, Y., Bomba, H.N., Ye, Y., Jiang, T., Isaacson, A.J., Gu, Z., 2016. Tumor microenvironment-medicated construction and deconstruction of extracellular drug-delivery depots. Nano Lett. 16, 1118-1126.

Huang, C.C., Chia, W.T., Chung, M.F., Lin, K.J., Hsiao, C.W., Jin, C., Lim, W.H., Chen, C.C., Sung, H.W., 2016. An implantable depot that can generate oxygen in situ for overcoming hypoxia-induced resistance to anticancer drugs in chemotherapy. J. Am. Chem. Soc. 138, 5222-5225.

Huang, Z.M., He, C.L., Yang, A., Zhang, Y., Han, X.J., Yin, J., Wu, Q., 2006. Encapsulating drugs in biodegradable ultrafine fibers through co-axial electrospinning. J. Biomed. Mater. Res. A,77, 169-179.

Illangakoon, U.E., Yu, D.G., Ahmad, B.S., Chatterton, N.P., Williams, G.R., 2015. 5-Fluorouracil loaded Eudragit fibers prepared by electrospinning. Int. J. Pharm. 495, 895-902. 
Ji, X., Wang, T., Guo, L., Xiao, J., Li, Z., Zhang, L., Deng, Y., He. N., 2013. Effect of nanoscale- $\mathrm{ZnO}$ on the mechanical property and biocompatibility of electrospun poly (L-lactide) acid/nanoscale-ZnO mats. J. Biomed. Nanotechnol. 9, 417-423.

Ji, X., Yang, W., Wang, T., Mao, C., Guo, L., Xiao, J., He. N., 2013. Coaxially electrospun core/shell structured poly (L-lactide) acid/chitosan nanofibers for potential drug carrier in tissue engineering. J. Biomed. Nanotechnol. 9, 1672-1678.

Jin, E.M., Park, J.Y., Zhao, X.G., Lee, I.H., Jeong, S.M., Gu, H.B., 2014. Photovoltaic properties of $\mathrm{TiO}_{2}-\mathrm{ZrO}_{2}$ fiber composite electrodes for dye-sensitized solar cells. Mater. Lett. 126, 281-284.

Joye, I.J., Nelis, V.A., McClements, D.J., 2015. Gliadin-based nanoparticles: Fabrication and stability of food-grade colloidal delivery systems. Food Hydrocoll. 44, 86-93.

Kazemimostaghim, M., Rajkhowa, R., Wang, X.G., 2015. Drug loading and release studies for milled silk particles of different sizes. Powder Technol. 283, 321-327.

Kim, D.Y., Kwon, D.Y., Kwon, J.S., Park, J.H., Park, S.H., Oh, H.J., Kim, J.H., Min, B.H., Park, K., Kim, M.S., 2016. Synergistic anti-tumor activity through combinational intratumoral injection of an in-situ injectable drug depot. Biomaterials 85, 232-245.

Li, W., Yu, D.G., Chen, K., Wang, G., Williams, G.R., 2013. Smooth preparation of ibuprofen/zein microcomposites using an epoxy-coated electrospraying head. 
Mater. Lett. 93, 125-128.

Liu, Y., Feng, N.P., 2015. Nanocarriers for the delivery of active ingredients and fractions extracted from natural products used in traditional Chinese medicine (TCM). Adv. Colloid Interface Sci. 221, 60-76.

Muehlenfeld, C., Kann, B., Windbergs, M., Thommes, M., 2013. Solid dispersion prepared by continuous cogrinding in an air jet mill. J. Pharm. Sci. 102, 4132-4139.

Paaver, U., Heinämäki, J., Laidmäe, I., Lust, A., Kozlova, J., Sillaste, E., Kirsimäe, K. Veski, P., Kogermann, K., 2015. Electrospun nanofibers as a potential controlled-release solid dispersion system for poorly water-soluble drugs. Int. J. Pharm. 479, 252-260.

Paliwal, R., Palakurthi, S., 2014. Zein in controlled drug delivery and tissue engineering. J. Control. Release 189, 108-122.

Pascariu, P., Airinei, A., Olaru, N., Olaru, L., Nica, V., 2016. Photocatalytic degradation of Rhodamine $\mathrm{B}$ dye using $\mathrm{ZnO}-\mathrm{SnO}_{2}$ electrospun ceramic nanofibers. Ceram. Int. 42, 6775-6781.

Pelipenko, J., Kocbek, P., Kristl, J., 2015. Critical attributes of nanofibers: preparation, drug loading, and tissue regeneration. Int. J. Pharm. 484, 57-74.

Peltonen, L., Valo, H., Kolakovic, R., Laaksonen, T., Hirvonen, J., 2010. Electrospraying, spray drying and related techniques for production and 
formulation of drug nanoparticles. Expert Opin. Drug Deliv. 7, 705-719.

Peppas, N.A., 1985. Analysis of Fickian and non-Fickian drug release from polymers. Pharm. Acta Helv. 60, 110-111.

Puncochova, K., Vukosavljevic, B., Hanus, J., Beranek, J., Windbergs, M., Stepanek, F., 2016. Non-invasive insight into the release mechanisms of a poorly soluble drug from amorphous solid dispersions by confocal Raman microscopy. Eur. J. Pharm. Biopharm. 101, 119-125.

Rasekh, M., Young, C., Roldo, M., Lancien, F., Le Mevel, J.C., Hafizi, S., Ahmad, Z., Barbu, E., Gorecki, D., 2015. Hollow-layered nanoparticles for therapeutic delivery of peptide prepared using electrospraying. J. Mater. Sci. Mater. Med. 26, 256.

Reddy, N., Yang, Y.Q, 2008. Self-crosslinked gliadin fibers with high strength and water stability for potential medical applications. J. Mater. Sci.: Mater. Med. 19, 2055-2061.

Rosic, R., Pelipenko, J., Kristl, J., Kocbeck, P., Baumgartner, S., 2012. Properties, engineering and applications of polymericNanofibers: Current research and future advances. Chem. Biochem. Eng. Q., 26, 417-425.

Saeidpour, S., Lohan, S.B., Solik, A., Paul, V., Bodmeier, R., Zoubari, G., Bittl, R., Meinke, M.C., Teutloff, C., 2017. Drug Distribution in Nanostructured Lipid Particles. Eur. J. Pharm. Biopharm. 110, 19-23. 
Sarmah, M., Banik, N., Hussain, A., Ramteke, A., Sharma, H.K., Maji, T.K., 2015. Study on crosslinked gelatin-montmorillonite nanoparticles for controlled drug delivery applications. J. Mater. Sci. 50, 7303-7313.

Schrank, S., Kann, B., Saurugger, E., Hainschitz, M., Windbergs, M., Glasser, B.J., Khinast, J., Roblegg, E., 2015. The effect of the drying temperature on the properties of wet-extruded calcium stearate pellets: pellet microstructure, drug distribution, solid state and drug dissolution. Int. J. Pharm. 478, 779-787.

Seif, S., Franzen, L., Windbergs, M., 2015. Overcoming drug crystallization in electrospun fibers--Elucidating key parameters and developing strategies for drug delivery. Int. J. Pharm. 478, 390-397.

Shutava, T.G., Fakhrullin, R.F., Lvov, Y.M., 2014. Spherical and tubule nanocarriers for sustained drug release. Curr Opin Pharmacol 18, 141-148.

Shin, D., Lee, S.J., Ha, Y.-M., Choi, Y.-S., Kim, J.-W., Park, S.-R., Park, M.K., 2017. Pharmacokinetic and pharmacodynamic evaluation according to absorption differences in three formulations of ibuprofen. Drug Des., Dev. Ther., 11, 135.

Soares, R.M.D., Patzer, V.L., Dersch, R., Wendorff, J., da Silveira, N.P., Pranke, P., 2011. A novel globular protein electrospun fiber mat with the addition of polysilsesquioxane. Int. J. Biol. Macromol. 49, 480-486.

Sóti, P.L., Nagy, Z.K., Serneels, G., Vajna, B., Farkas, A., Van der Gucht, F., Fekete, P., Vigh, T., Wagner, I., Balogh, A., Pataki, H., Mező, G., Marosi, G., 2015. Preparation and comparison of spray dried and electrospun bioresorbable drug 
delivery systems. Eur. Polym. J. 68, 671-679.

Taha, A.A., Wu, Y.N., Wang, H.T., Li, F.T., 2012. Preparation and application of functionalized cellulose acetate/silica composite nanofibrous membrane via electrospinning for $\mathrm{Cr}(\mathrm{VI})$ ion removal from aqueous solution. J. Environ. Manage. 112, 10-16.

Tolosa, A., Krüner, B., Jäckel, N., Aslan, M., Vakifahmetoglu, C., Presser, V., 2016. Electrospinning and electrospraying of silicon oxycarbide-derived nanoporous carbon for supercapacitor electrodes. J. Pow. Sour. 313, 178-188.

Vukosavljevic, B., De Kinder, L., Siepmann, J., Muschert, S., Windbergs, M., 2016. Novel insights into controlled drug release from coated pellets by confocal Raman microscopy. J. Raman Spectrosc. 47, 757-762.

Wan, F., Maltesen, M.J., Andersen, S.K., Bjerregaard, S., Foged, C., Rantanen, J., Yang. M., 2014. One-step production of protein-loaded PLGA microparticles via spray drying using 3-fluid nozzle. Pharm. Res. 31, 1967-1977.

Wan, Z.L., Guo, J., Yang, X.Q., 2015. Plant protein-based delivery systems for bioactive ingredients in foods. Food Funct 6, 2876-2889.

Wang, T., Ji, X., Jin, L., Feng, Z., Wu, J., Zheng, J., Wang, H., Xu, Z.-W., Guo, L., He. N., 2013, Fabrication and characterization of heparin-grafted poly-L-lactic acid-chitosan core-shell nanofibers scaffold for vascular gasket. ACS Appl. Mater. Interfaces 5, 3757-3763. 
Wang, X., Yu, D.G., Li, X.Y., Bligh, S.A., Williams, G.R., 2015. Electrospun medicated shellac nanofibers for colon-targeted drug delivery. Int. J. Pharm. 490, 384-390.

Wang, Y.X., Chen, L.Y., 2012. Electrospinning of prolamin proteins in acetic acid: The effects of protein conformation and aggregation in solution. Macromol. Mater. Eng. 297, 902-913.

Wen, H.F., Yang, C., Yu, D.G., Li, X.Y., Zhang, D.F., 2016. Electrospun zein nanoribbons for treatment of lead-contained wastewater. Chem. Eng. J. 290, 263-272.

Windbergs, M., Haaser, M., McGoverin, C.M., Gordon, K.C., Kleinebudde, P., Strachan, C.J., 2010. Investigating the relationship between drug distribution in solid lipid matrices and dissolution behaviour using Raman spectroscopy and mapping. J. Pharm. Sci. 99, 1464-1475.

Xu, F., Xu, Y., Yu. D., 2015. Zero-order controlled release nanofibers fabricated using coaxial electrospinning with polymer dilute solution as a sheath fluid. J. Uni. Shanghai Sci. Technol. 37, 165-168.

Yang, C., Yu, D.G., Pan, D., Liu, X.K., Wang, X., Bligh, S.W.A., Williams, G.R., 2016. Electrospun $\mathrm{pH}$-sensitive core-shell polymer nanocomposites fabricated using a tri-axial process. Acta Biomater. 35, 77-86.

Yang, G.Z., Li, J.J., Yu, D.G., He, M.F., Yang, J.H., Williams G.R., 2017. Nanosized sustained-release drug depots fabricated using modified tri-axial electrospinning. 
Acta Biomater. 53, 233-241.

Yu, D.G., Yu, J.H., Chen, L., Williams, G.R., Wang, X., 2012. Modified coaxial electrospinning for the preparation of high-quality ketoprofen-loaded cellulose acetate nanofibers. Carbohydr. Polym. 90, 1016-1023.

Yu, D.G., Li, X.Y., Wang, X., Yang, J.H., Bligh, S.W.A., Williams, G.R., 2015. Nanofibers fabricated using triaxial electrospinning as zero order drug delivery systems. ACS Appl. Mater. Interfaces 7, 18891-18897.

Yu, D.G., Li, J.J., Zhang, M., Williams, G.R., 2017. High-quality Janus nanofibers prepared using three-fluid electrospinning. Chem. Commun. 53, 4542-4545.

Zhang, Y., Price, G.W., Jamieson, R., Burton, D., Khosravi, K., 2017. Sorption and desorption of selected non-steroidal anti-inflammatory drugs in an agricultural loam-textured soil. Chemosphere, 174, 628-637.

Zhu, J.D., Lu, Y., Chen, C., Ge, Y.Q., Jasper, S., Leary, J.D., Li, D.W., Jiang, M.J., Zhang, X.W., 2016. Porous one-dimensional carbon/iron oxide composite for rechargeable lithium-ion batteries with high and stable capacity. J. Alloys Compd. $672,79-85$.

\section{Table and Figure legends}


Table 1. Experimental parameters for the fabrication of IBU-loaded fibers.

Fig. 1. SEM images of electrospun gliadin fibers prepared from solutions with different concentrations in HFIP: (a) 5\%, (b) $10 \%$, (c) $15 \%$, and (d) $20 \%$.

Fig. 2. Surface SEM images of raw materials and drug-loaded fibers. (a) IBU, (b) gliadin; (c) M1, (d) M2, (e) E1, and (f) E2.

Fig. 3. SEM images of the cross-section of drug-loaded fibers. (a) M1, (b) M2, (c) E1 and (d) E2.

Fig. 4. X-ray diffraction patterns of (a) IBU, (b) gliadin (c) M1, (d) M2, (e) E1 and (f) E2.

Fig. 5. (a) IR spectra of the raw materials and drug-loaded fibers, together with (b) the molecular structures of IBU and gliadin, and (c) a schematic showing possible hydrogen bonding interactions.

Fig. 6. Raman spectra of (a) IBU, (b) gliadin, (c) M1, (d) M2, (e) E1, and (f) E2.

Fig. 7. In vitro drug release data. (a) IBU release as a function of time ( $\mathrm{n}=6$; data reported as mean \pm S.D.), and (b) the time needed to release various percentages of the IBU content.

Fig. 8. Schematic diagrams showing (a) the proposed formation mechanism of the core/shell fibers from the traditional coaxial process, and (b) the drug distributions within fibers prepared from the different coaxial spinning modalities. 
Table 1. Experimental parameters for the fabrication of IBU-loaded fibers.

${ }^{a}$ These values are theoretically calculated according to the compositions and flow rates of the working fluids.

\begin{tabular}{|c|c|c|c|c|c|}
\hline & Process & $\begin{array}{l}\text { Core fluid } \\
\text { composition } \\
(\mathrm{w} / \mathrm{v})\end{array}$ & $\begin{array}{l}\text { Sheath fluid } \\
\text { composition } \\
(\mathrm{w} / \mathrm{v})\end{array}$ & $\begin{array}{l}\text { Core/sheath } \\
\text { fluid flow rates } \\
(\mathrm{mL} / \mathrm{h})\end{array}$ & $\begin{array}{l}\text { Drug content in } \\
\text { fibers }(\%)^{\mathrm{a}}\end{array}$ \\
\hline M1 & \multirow{2}{*}{$\begin{array}{l}\text { Modified } \\
\text { coaxial }\end{array}$} & $\begin{array}{c}1 \% \text { IBU } \\
15 \% \text { gliadin }\end{array}$ & HFIP & $2.5 / 0.5$ & 6.25 \\
\hline M2 & & $\begin{array}{l}2 \% \text { IBU } \\
15 \% \text { gliadin }\end{array}$ & HFIP & $2.5 / 0.5$ & 11.76 \\
\hline E1 & \multirow{2}{*}{$\begin{array}{l}\text { Traditional } \\
\text { coaxial }\end{array}$} & $5 \%$ IBU & $15 \%$ gliadin & $0.5 / 2.5$ & 6.25 \\
\hline E2 & & $10 \%$ IBU & $15 \%$ gliadin & $0.5 / 2.5$ & 11.76 \\
\hline
\end{tabular}



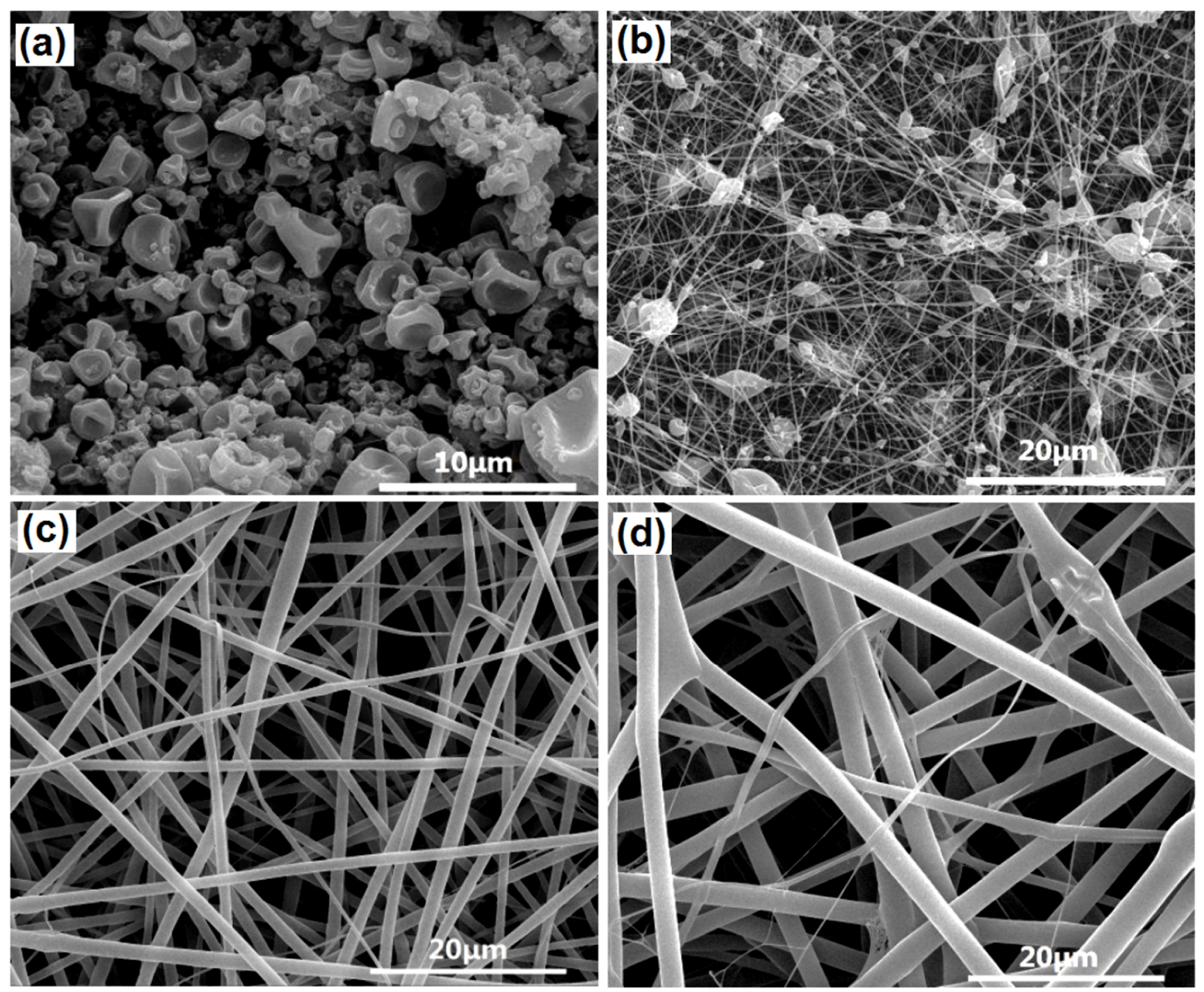

Fig. 1. SEM images of electrospun gliadin fibers prepared from solutions with different concentrations in HFIP: (a) 5\%, (b) 10\%, (c) 15\%, and (d) $20 \%$. 


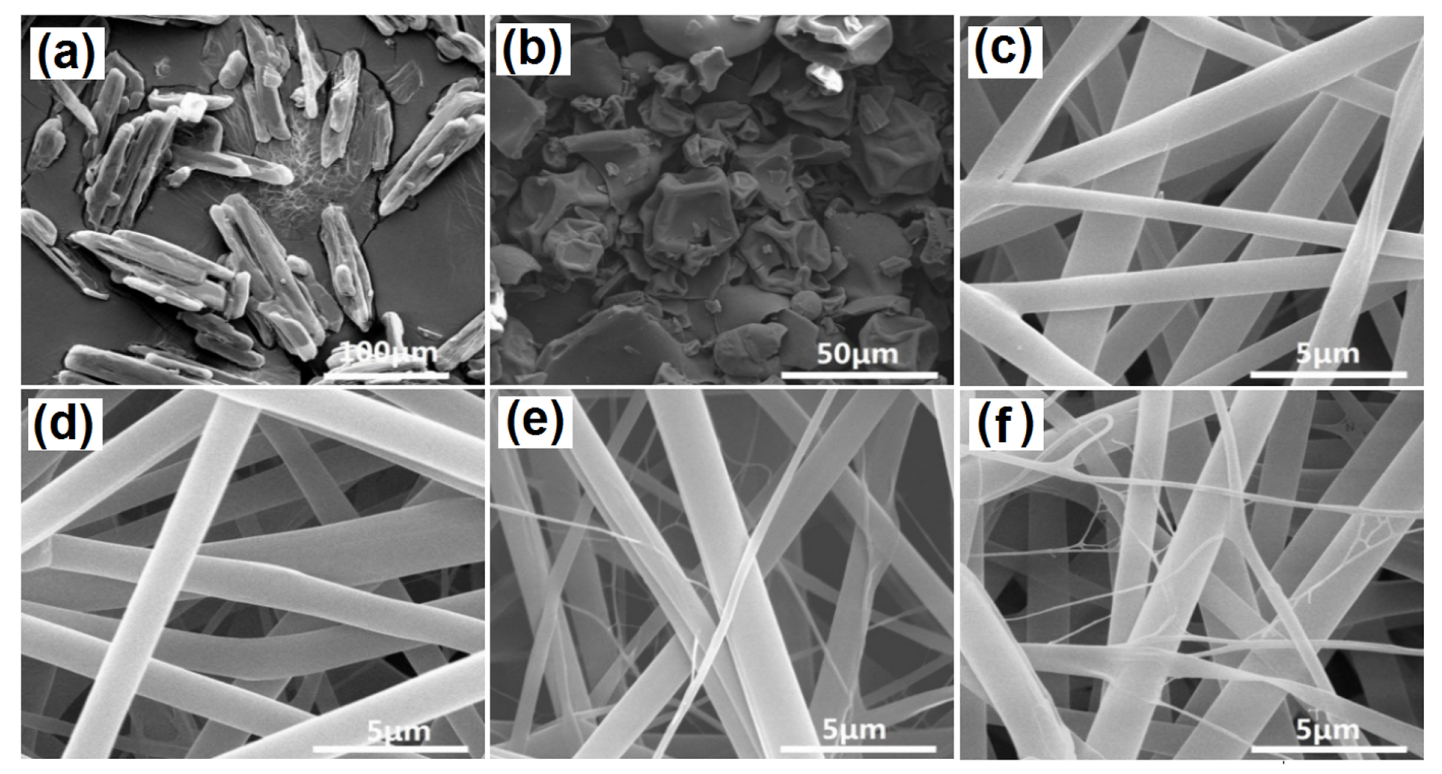

Fig. 2. Surface SEM images of raw materials and drug-loaded fibers. (a) IBU, (b) gliadin; (c) M1, (d) M2, (e) E1, and (f) E2. 

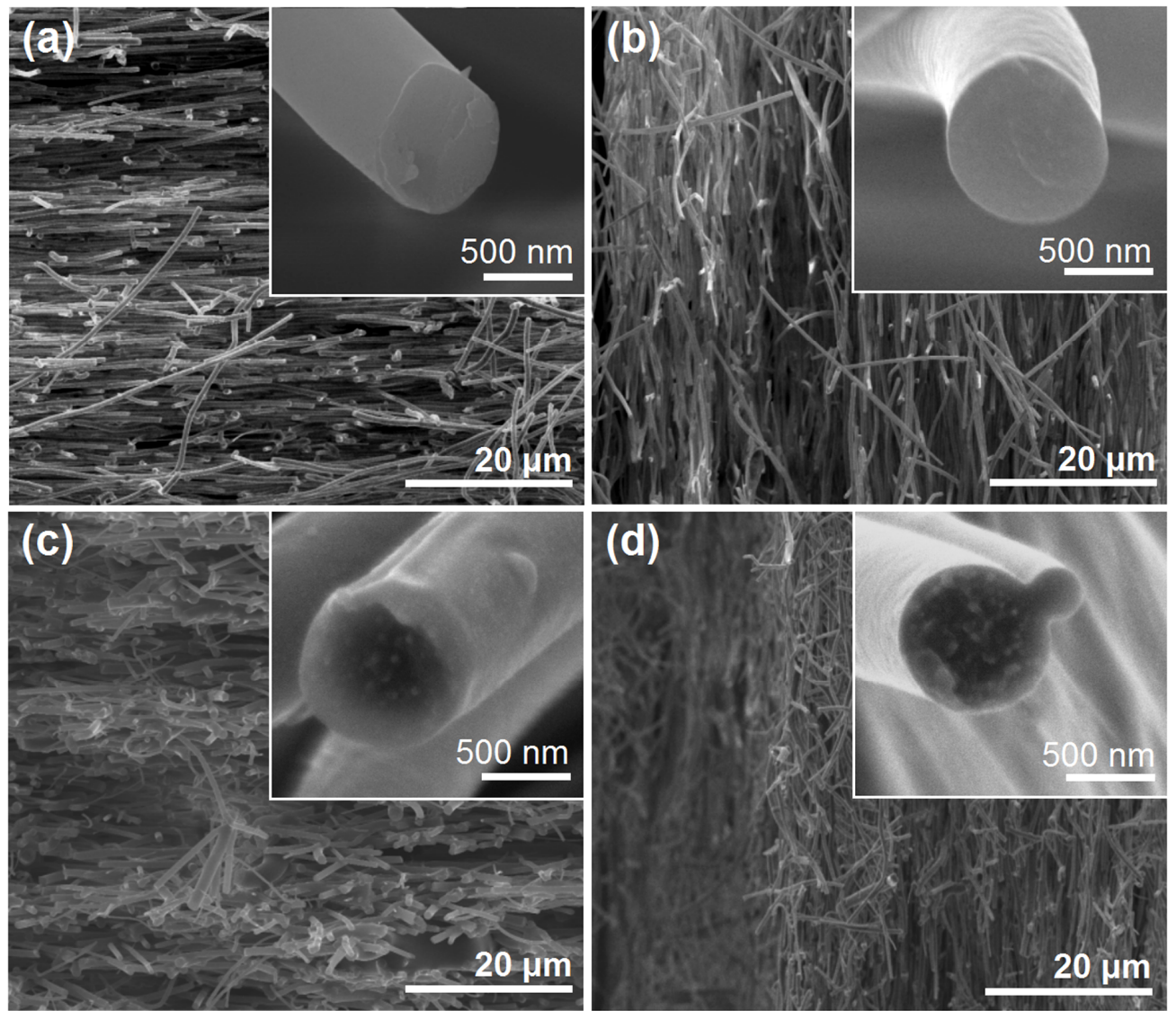

Fig. 3. SEM images of the cross-section of drug-loaded fibers. (a) M1, (b) M2, (c) E1 and (d) E2. 


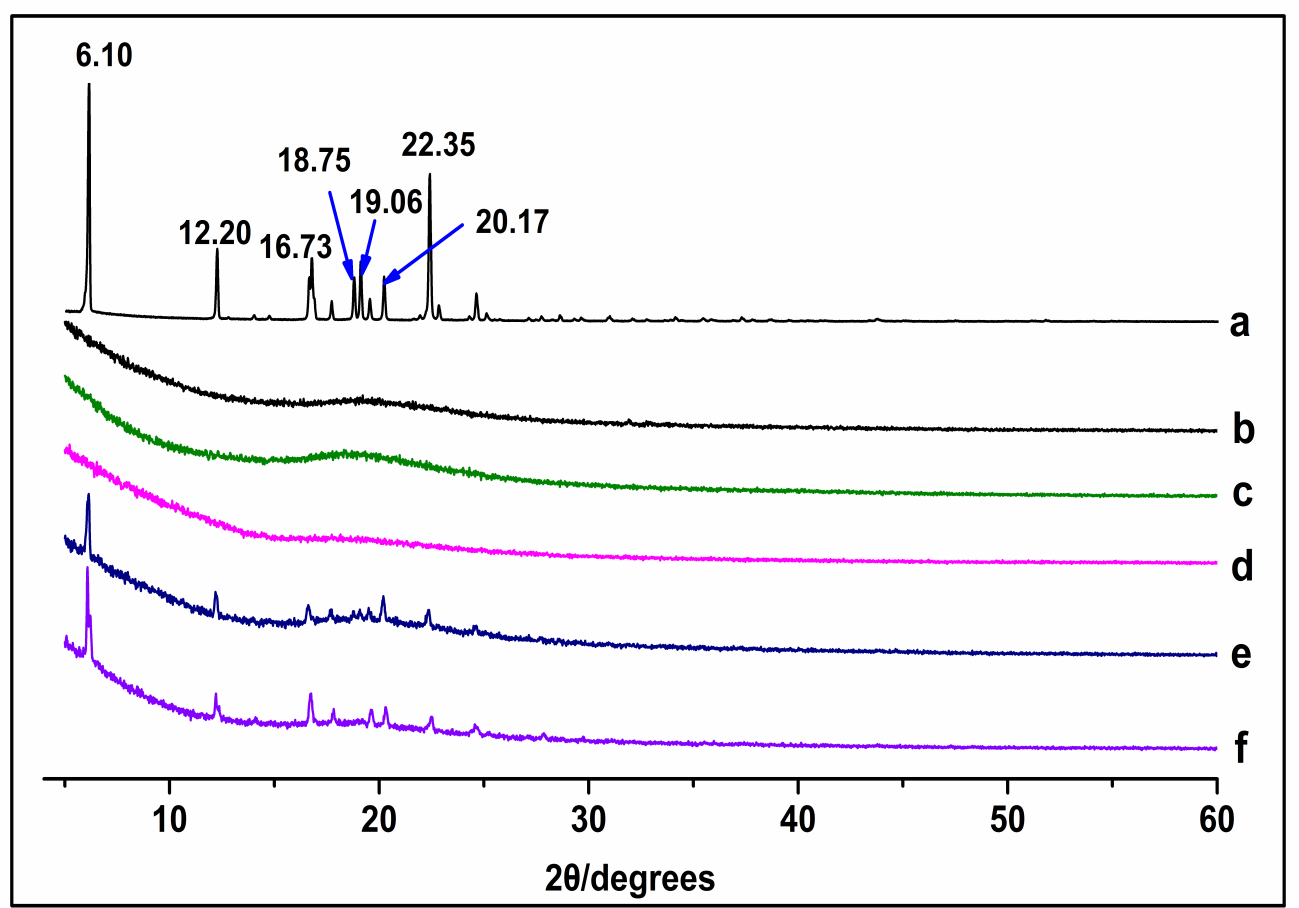

Fig. 4. X-ray diffraction patterns of (a) IBU, (b) gliadin (c) M1, (d) M2, (e) E1 and (f) E2. 


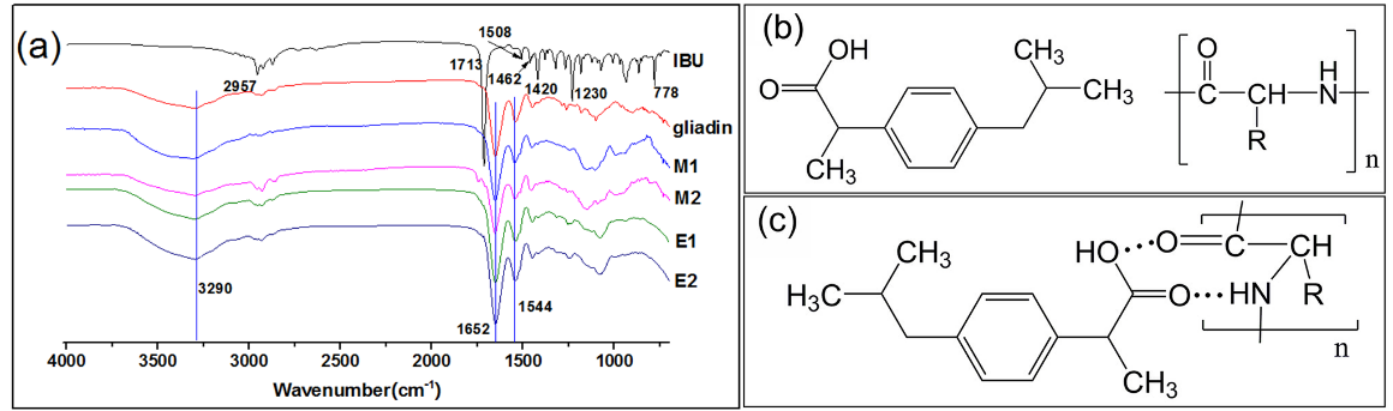

Fig. 5. (a) IR spectra of the raw materials and drug-loaded fibers, together with (b) the molecular structures of IBU and gliadin, and (c) a schematic showing possible hydrogen bonding interactions. 


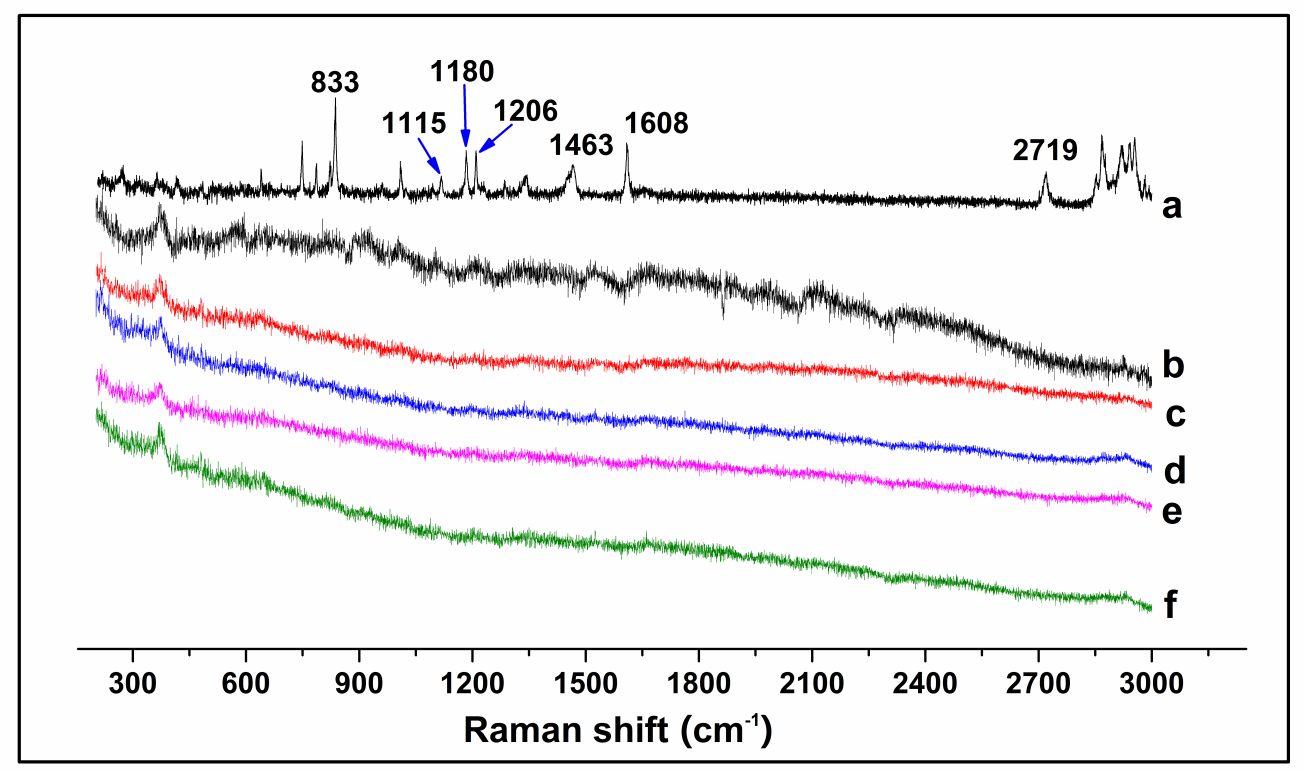

Fig. 6. Raman spectra of (a) IBU, (b) gliadin, (c) M1, (d) M2, (e) E1, and (f) E2. 

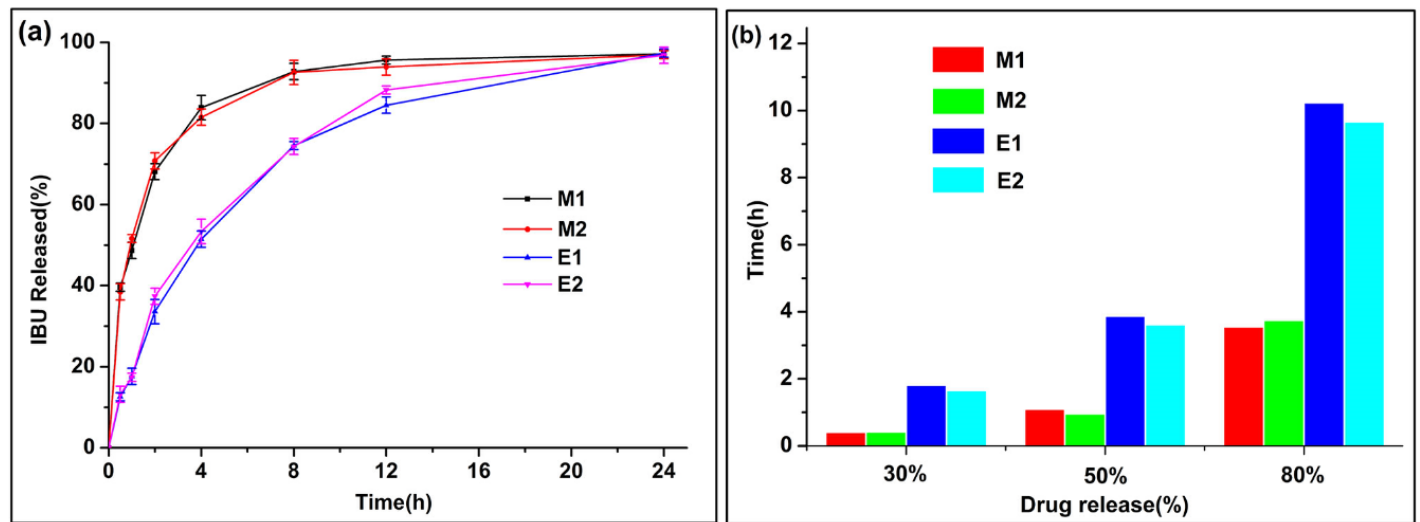

Fig. 7. In vitro drug release data. (a) IBU release as a function of time ( $\mathrm{n}=6$; data reported as mean \pm S.D.), and (b) the time needed to release various percentages of the IBU content. 


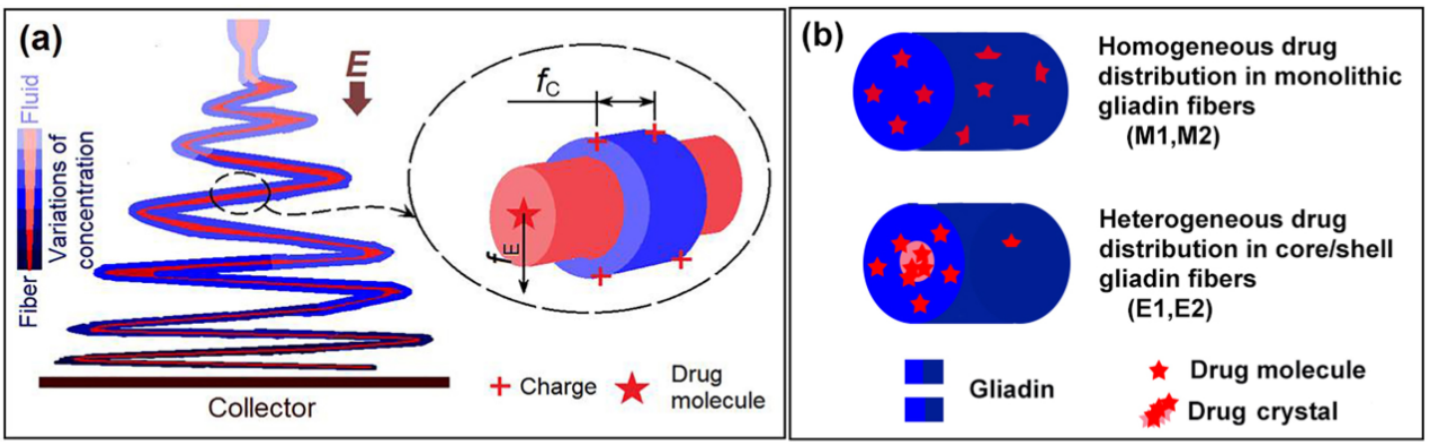

Fig. 8. Schematic diagrams showing (a) the proposed formation mechanism of the core/shell fibers from the traditional coaxial process, and (b) the drug distributions within fibers prepared from the different coaxial spinning modalities. 